\title{
High-performance Grating Coupled Surface Plasmon Resonance Sensor Based on Al-Au Bimetallic Layer
}

\author{
Changkui Hu (Corresponding author) \\ School of Optoelectronics Science and Engineering \\ Huazhong University of Science and Technology, Wuhan 430074, China \\ $\&$ \\ School of science, Wuhan University of Technology, Wuhan 430070, China \\ Tel: 86-27-8765-5750Ｅ-mail: hck@whut.edu.cn \\ Deming Liu \\ School of Optoelectronics Science and Engineering \\ Huazhong University of Science and Technology, Wuhan 430074, China \\ Tel: 86-27-8755-6188Ｅ-mail: dmliu@mail.hust.edu.cn
}

\begin{abstract}
A high-performance grating coupled surface plasmon resonance (GCSPR) sensor based on Al-Au bimetallic layer is proposed. High sensitivity is obtained by replacing the positive diffraction order with negative diffraction order of metallic grating to excite the surface plasmon. Compared with the conventional Au-based GCSPR sensor, the sensor with aluminum as the SPR active metal exhibits a sharper (i.e. larger depth-to-width ratio) reflectivity dip, which increases the signal-to-noise ratio (SNR) and enhances its resolution. In addition, an ultrathin gold film is deposited on the grating surface in order to protect the Al layer from getting oxidized. Numerical simulations show that the angular sensitivity of the sensor reaches $187.2^{\circ}$ RIU (degree per refractive index unit) with good linearity and the FWHM (full width at half maximum) of the resonant dip is only $0.93^{\circ}$.
\end{abstract}

Keywords: Surface plasmons, Optical sensing and sensors, Gratings

\section{Introduction}

Surface plasmon resonance (SPR) has been widely used in a variety of sensing applications, since it provides high sensitivity, label-free, real-time and rapid sensing capability for detecting biomedical and biochemical reactions on a surface. (Nylander et al., 1982, p.79; Matsubara et al., 1988, p.1160; Homola, 1999, p.3). Generally, the SPR sensor structures can be based on either attenuated total reflection (ATR) prism coupling or metallic grating coupling. However, the prism-based systems are widely used in practice because their sensitivity is 2-3 times higher than the grating-based systems. (Cullen et al.,1990,p.576). Recently, many researches on improving the sensitivity of grating-based system are reported. Byun's group proposed a grating-coupled transmission-type SPR sensor with a refractive index sensitivity of $70^{\circ} /$ RIU by resonant coupling surface plasmon to radiation modes. (Byun et al., 2007, p.5703). The grating-based systems' sensitivities are reported as $97^{\circ} / \mathrm{RIU}$ in a typical single-mode method, $103^{\circ} / \mathrm{RIU}$ in a short-range mode method. (Guo et al., 2008, p.512). All the grating-based methods above are improving the sensitivity through making the dips of the 1st diffraction order better, which can not exceed the sensitivity of the prism-based systems. Moreover, from the report of Cai (2008,p.14597), the refractive index sensitivity of grating-based SPR sensors is improved into more than $237^{\circ} /$ RIU by double-dips method. However, according to his report, the price paying for this sensitivity enhancement is the additional uncertainty of estimating two spectral shifts instead of one in the conventional methods based on single resonant dip.

In this paper, we present a grating coupled surface plasmon resonance sensor with aluminum as the active metal. Rigorous coupled wave analysis (RCWA) (Moharam et al., 1986, p.1780) is applied to study the influences of the grating period and the refractive index of sensing layer on the resonant properties. Based on the analysis, high refractive index sensitivity is obtained by exciting the surface plasmon with -1st diffraction order of metallic grating. Compared with the conventional Au-based GCSPR sensor, the sensor with aluminum as the SPR active metal exhibits a sharper reflectivity dip, which increases the signal-to-noise ratio (SNR) and 
enhances its resolution. In addition, the oxidation problem of an Al-based GCSPR sensor has been addressed by coating it with an ultrathin gold layer. The physical explanation related to the results has been provided.

\section{Numerical Model}

Figure 1 shows a schematic diagram of the grating coupled SPR sensor model. In this geometry, the momentum of the incident wave can be increased to match that of a surface plasmon wave by diffraction at a rectangle metallic grating. The grooves of metallic grating are oriented perpendicular to the incident plane of TM light. $\Lambda$ is the period of metallic grating, $W$ is the ridge width of grating, $f=W / \Lambda$ is the duty cycle, and $h$ is the depth of grating. Assuming the dispersion properties of SPW are not disturbed by the grating, the momentum conservation for an optical wave exciting an SPW via a diffraction grating may be expressed as: (Homola et al., 1999, p.16).

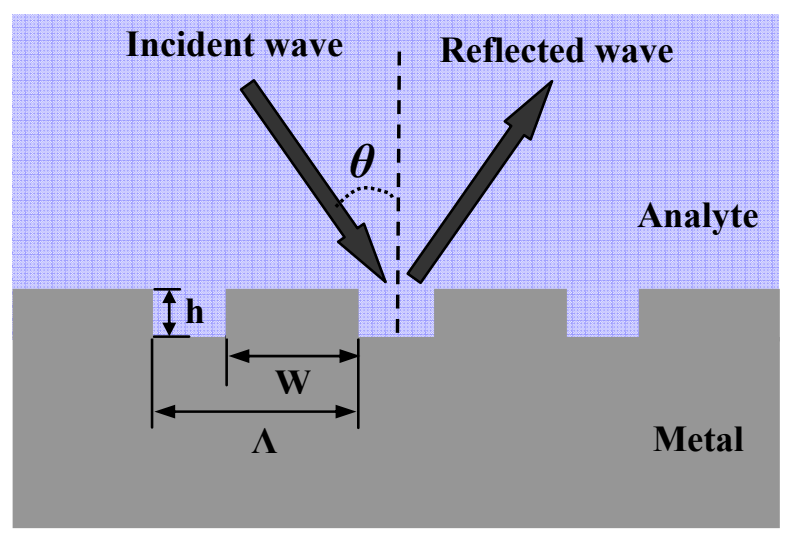

Figure 1. Schematic Diagram of the Metallic Grating Coupled SPR Sensor

$$
n_{a} \sin \theta_{R}+m \frac{\lambda}{\Lambda}= \pm \sqrt{\frac{\varepsilon_{m} n_{a}^{2}}{\varepsilon_{m}+n_{a}^{2}}}
$$

where $\theta_{R}$ is the resonant angle of incidence, $\varepsilon_{m}\left(\varepsilon_{m}=\varepsilon_{m r}+i \varepsilon_{m i}\right)$ is the permittivity of metal, $n_{a}$ is the refractive index of the dielectric (analyte). Sign ' + ' and sign '-' correspond to diffracted waves of orders $m>0$ and $m<0$ respectively. Equation (1) indicates that the resonant angle $\theta_{R}$ is a monotonic function of refractive index $n_{a}$, therefore, the configurations can perform as a refractive index sensor in angular interrogation and the refractive index sensitivity $S$ is defined as the ratio of the shift in resonant angle $\left(\delta \theta_{R}\right)$ to the change in the refractive index of the dielectric $\left(\delta n_{a}\right)$.

Effectively excitation of surface plasmon generally requires $\left|\varepsilon_{m}\right|>>n_{a}^{2}$, therefore, the resonant angle is calculated from Eq. (1) as following:

$$
\sin \theta_{R}= \pm 1-m \cdot \frac{\lambda}{\Lambda \cdot n_{a}}
$$

The limitation on period of the metallic grating for both positive and negative diffraction order can be expressed as: (Lin et al, 2008, p.18599).

$$
\begin{array}{ll}
\frac{\Lambda}{\lambda}>\frac{m}{n_{a}} & m>0 \\
\frac{|m|}{2 n_{a}}<\frac{\Lambda}{\lambda}<\frac{|m|}{n_{a}} & m<0
\end{array}
$$

The conditions on period of grating for several lower order diffractions can be achieved from Eq. (3) as 
following:

$$
\begin{aligned}
& \frac{1}{2 n_{a}}<\frac{\Lambda}{\lambda}<\frac{1}{n_{a}}, \quad m=-1 ; \\
& \frac{1}{n_{a}}<\frac{\Lambda}{\lambda}<\frac{3}{2 n_{a}}, \quad m=1,-2 ; \\
& \frac{3}{2 n_{a}}<\frac{\Lambda}{\lambda}<\frac{2}{n_{a}}, \quad m=1,-2,-3 ; \\
& \frac{2}{n_{a}}<\frac{\Lambda}{\lambda}<\frac{5}{2 n_{a}}, \quad m=1,2,-3,-4 .
\end{aligned}
$$

\section{Results and Discussion}

Using RCWA method, the angular reflection spectrum of the grating coupled SPR sensor with aluminum as the active metal are calculated for different periods of $\Lambda=1400 \mathrm{~nm}, 1100 \mathrm{~nm}, 700 \mathrm{~nm}$, and $350 \mathrm{~nm}$, as shown in Figure 2, the operating wavelength is $900 \mathrm{~nm}$, the depth and duty cycle of the grating are $30 \mathrm{~nm}$ and 0.9 respectively, and the permittivity of aluminum is determined through Drude model. (Jha et al., 2009, p.759).
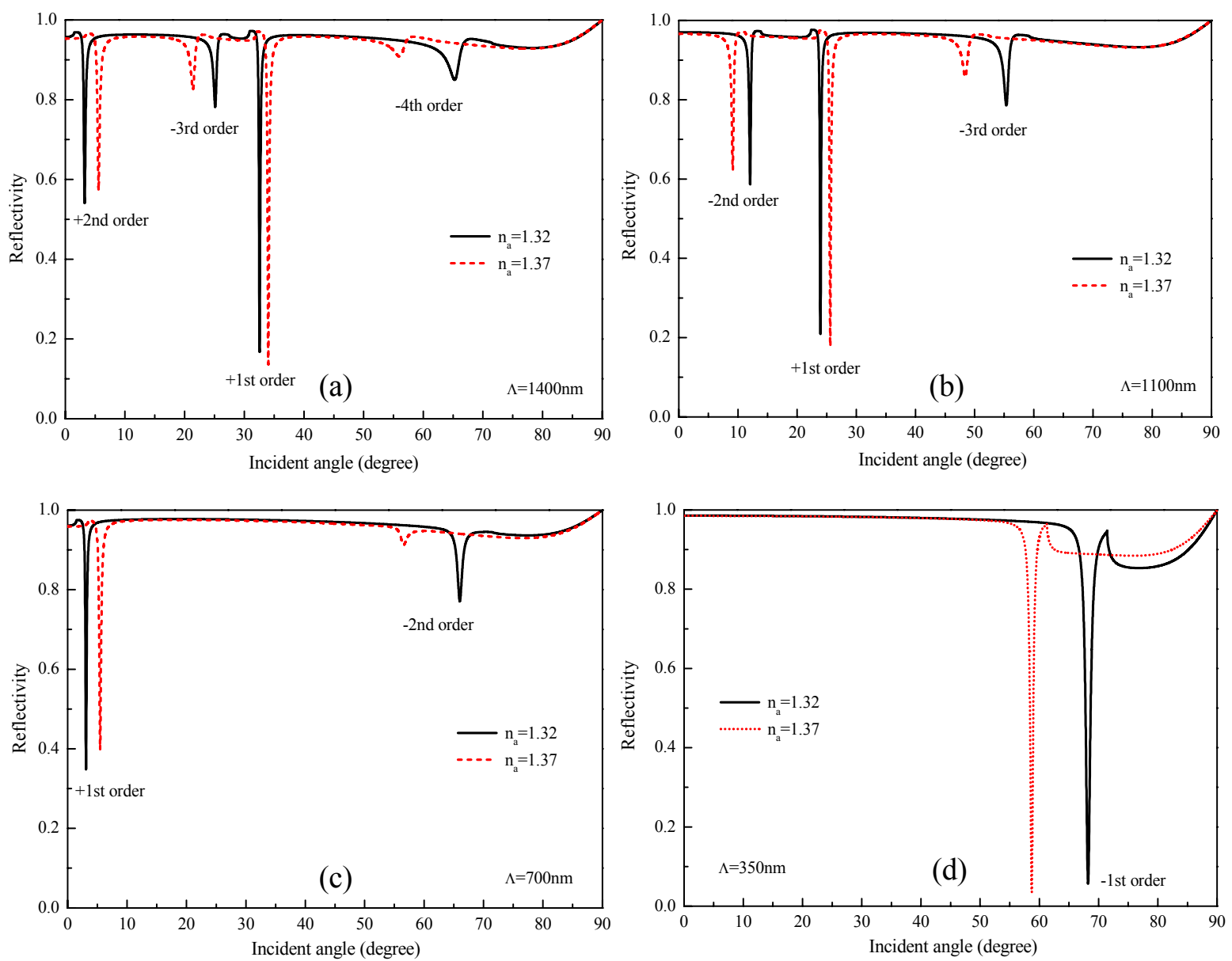

Figure 2. Reflectivity as a function of the incident angle calculated for two different refractive indices of analyte and four different grating periods: (a) $\Lambda=1400 \mathrm{~nm}$, (b) $\Lambda=1100 \mathrm{~nm}$, (c) $\Lambda=700 \mathrm{~nm}$, (d) $\Lambda=350 \mathrm{~nm}$.

As the refractive index of analyte $\left(n_{a}\right)$ increases, resonant angle $\theta_{R}$ goes to bigger for positive diffraction order and becomes smaller for negative diffraction order, which is identical with the Eq. (2). In addition, it can be seen that the refractive index sensitivity using negative diffraction order dip is larger than that using the positive diffraction order dip. Taking into account both the shift in resonant angle and the depth-width ratio of 
the SPR curve, we apply -1 diffraction order of the metallic grating into sensoring and introduce the quality parameter $\chi$ defined by $\chi=S / w$ to denote the overall performance of sensor in the following discussion, where $S$ and $w$ are the refractive index sensitivity and the FWHM (Full Width at Half Maximum) of the SPR dip.

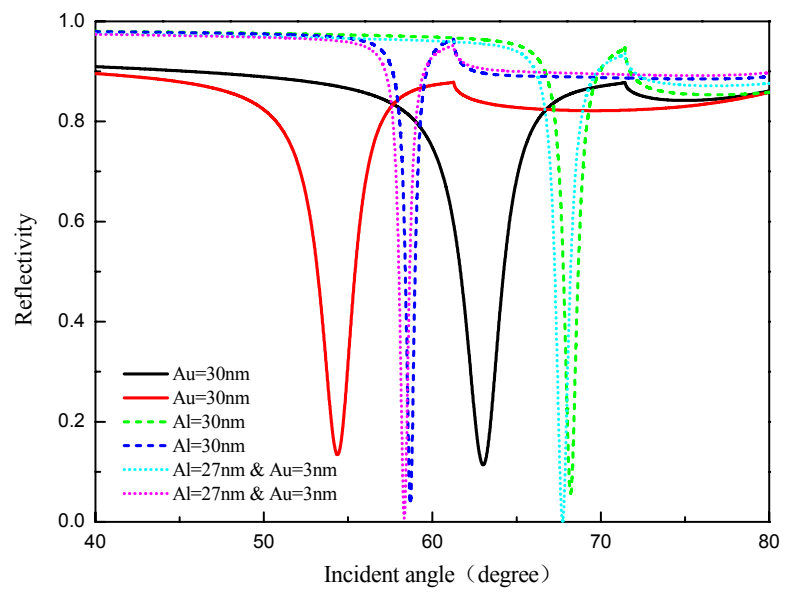

Figure 3. Angular SPR curves of the grating-based SPR sensor with two different refractive index of analyte for monolayer of $\mathrm{Au}(30 \mathrm{~nm}), \mathrm{Al}(30 \mathrm{~nm})$, and bimetallic layer $(27 \mathrm{~nm}$ of $\mathrm{Al}$ and $3 \mathrm{~nm}$ of $\mathrm{Au})$.

As the refractive index of analyte $\left(n_{a}\right)$ is 1.32 and 1.37 respectively, two SPR curves with Al and Au as the SPR active metal were shown in figure 3 . The operating wavelength is $900 \mathrm{~nm}$. The structure parameters of the grating are $\Lambda=350 \mathrm{~nm}, f=0.9$ and $h=30 \mathrm{~nm}$. The graph also contains the SPR curve for a bimetallic case with $\mathrm{Al}$ as the active metal and a 3 -nm-thick Au film coating on the surface of the grating. As the refractive index of analyte $\left(n_{a}\right)$ varies from 1.32 to 1.37 , resonant angle $\theta_{R}$ shifts from $63.02^{\circ}$ to $53.37^{\circ}$ in the case of Au, whereas for Al the resonant angle $\theta_{R}$ shifts from $68.21^{\circ}$ to $58.70^{\circ}$. On the other hand, the FWHM of the SPR dip for Au and $\mathrm{Al}$, respectively, are $2.02^{\circ}$ and $0.82^{\circ}$. This means that the quality parameter $\chi$ of the Al-base sensor is almost more as $273 \%$ compared with an Au-base one. The much smaller FWHM for Al can be attributed to the smaller value of modulus of $\varepsilon_{m r} / \varepsilon_{m i}$ at a given wavelength as compared to Au, which is the most widely used as SPR active metal. If one considers the bimetallic case (27nm of Al and $3 \mathrm{~nm}$ for $\mathrm{Au}$ ), the shift in the resonant angle $\delta \theta_{R}$ is $9.39^{\circ}$ with FWHM of $0.93^{\circ}$, which is close to the case of Al. Moreover, the Au film protects the Al layer from getting oxidized.

Figure 4 shows that, for the case of Al-Au bimetallic layer, the resonant angle shifts from $68.21^{\circ}$ to $58.70^{\circ}$ as the refractive index of the analyte varies from 1.32 to 1.37 in steps of 0.01 . From linear regression analyses, the shift is completely linear over the whole range of refractive indices with correlation coefficient $R=0.99828$ while the sensitivity, which indicates the slope of resonance angle over the refractive index, is $187.2^{\circ} /$ RUI. For the case of $\mathrm{Au}, \mathrm{Al}$ and $\mathrm{Au}-\mathrm{Al}$ bimetallic layer, the performance of the grating-based systems are compared in Table1. In addition, to show the recent results reported in this area, several concerned results reported in literature recently are also presented in Table 1. They are the results of grating-coupled transmission-type SPR sensor (GC-TT) reported by Byun (2007,p.5703), and the results of grating-based systems in a short-range method (SRM) and typical single-mode method (SMM) reported by Guo (2008,p.512) and a double-dips method (DDM) reported by Cai (2008,p.14597), and the results of prism-based system with BK7 prism and synthesized quartz prism reported by Gupta (2007,p.381) . 

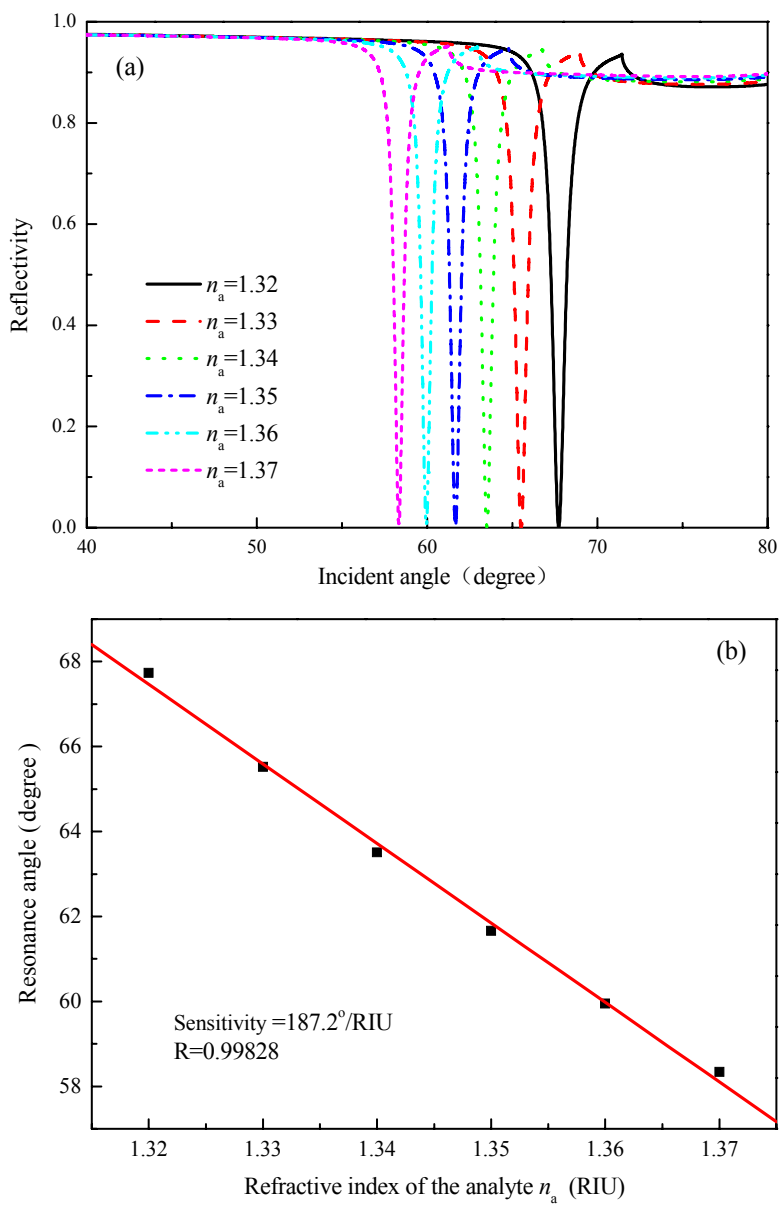

Figure 4. (a) Angular SPR curves of the grating-based SPR sensor with five different refractive indices of analyte. (b) Linear regression analysis between resonance angle and refractive indices of analyte.

Table 1. Comparison of the performance of different SPR sensors

\begin{tabular}{|c|c|c|c|c|c|c|c|c|c|}
\hline & \multicolumn{6}{|c|}{ Grating-based SPR } & \multicolumn{3}{c|}{ Prism-based SPR } \\
\cline { 2 - 10 } Feature & $\mathrm{Au}$ & $\mathrm{Al}$ & $\mathrm{Al}-\mathrm{Au}$ & GC-TT & SMM & SRM & DDM & BK7 prism & Synthesized quartz prism \\
\hline$S\left(^{\circ} / R I U\right)$ & 171 & 189.5 & 187.2 & 70 & 97 & 103 & 237 & 134.4 & 204.4 \\
\hline$F W H M\left(^{\circ}\right)$ & 2.02 & 0.82 & 0.93 & 1.35 & - & - & 2.5 & 3 & 4.36 \\
\hline$\chi\left(R I U^{-1}\right)$ & 85 & 231 & 201 & 52 & - & - & 95 & 47 & 47 \\
\hline
\end{tabular}

It can be seen that the sensitivity of grating coupled SPR sensor can be improved by using negative diffraction order of grating to excite the surface plasmon instead of positive diffraction order. Compared with the grating-based system in double-dips method and the prism-based system with synthesized quartz prism, the sensitivity of grating coupled SPR with Al-Au bimetallic is slightly lower, but its quality parameter $\chi$ is 2.12 times as the former, and 4.28 times as the later, which can be attributed to the smaller value of FWHM with $\mathrm{Al}$ as the SPR active metal. Taking both sensitivity and quality parameter into account, the grating-based SPR sensor with $\mathrm{Al}-\mathrm{Au}$ bimetallic layer has roundly better performance than others.

\section{Conclusion}

In conclusion, we present a high-performance grating coupled SPR sensor with Al-Au bimetallic layer. The 
influences of the period of metallic grating on SPR characteristics are investigated using RCWA. High sensitivity is obtained by using -1 diffraction order of the metallic grating to excite the surface plasmon. Compared with the conventional Au-based GCSPR sensor, the sensor with aluminum as the SPR active metal exhibits higher resolution. An ultrathin gold film deposited on the grating surface can protect the $\mathrm{Al}$ layer from getting oxidized. The results show that the angular sensitivity of the sensor reaches $187.2^{\circ}$ RIU with good linearity and good $\chi$ factors of $201 \mathrm{RIU}^{-1}$, which are better than the concerned results reported in literature recently.

\section{Acknowledgement}

This work was supported by the Pre-Research Special Project in Important Fundamental Research of China (Grant No. 2005CCA04200).

\section{References}

Byun, K. M., Kim, S. J., \& Kim, D. (2007). Grating-coupled transmission-type surface plasmon resonance sensors based on dielectric and metallic gratings. Appl. Opt., 46, 5703-5708.

Cai, D., Lu, Y., Lin, K., Wang, P., \& Ming, H. (2008). Improving the sensitivity of SPR sensors based on gratings by double-dips method (DDM). Opt. Express, 16, 14597-14602.

Cullen, D. C., \& Lowe, C. R. (1990). A direct surface plasmon-polariton immunosensor: Preliminary investigation of the non-specific adsorption of serum components to the sensor interface. Sens. Actuators B, 1, 576-579.

Guo, J., Keathley, P. D., \& Hastings, J. T. (2008). Dual-mode surface-plasmon-resonance sensors using angular interrogation. Opt. Lett., 33, 512-514.

Gupta, G., \& Kondon, J. (2007).Tuning and sensitivity enhancement of surface plasmon resonance sensor. Sens. Actuators B, 122, 381-388.

Homola, J., Koudela, I., \& Yee, S. S. (1999). Surface plasmon resonance sensors based on diffraction gratings and prism couplers: sensitivity comparison. Sens. Actuators B, 54, 16-24.

Homola, J., Yee, S. S., \& Gauglitz, G. (1999). Surface plasmon resonance sensors: review. Sens. Actuators B, 54, 3-15.

Jha, R., \& Sharma, A. K. (2009). High-performance sensor based on surface plasmon resonance with chalcogenide prism and aluminum for detection in infrared. Opt. Letters, 34, 749-751.

Lin, K., Lu, Y., Chen, J., Zheng, R., Wang, a P., \& Ming, H. (2008). Surface plasmon resonance hydrogen sensor based on metallic grating with high sensitivity. Opt. Express,16, 18599-18604.

Matsubara, K., Kawata, S., \& Minami, S. (1988). Optical chemical sensor based on surface plasmon measurement. Appl. Opt., 27, 1160-1163

Moharam M. G., \& T. K. Gaylord. (1986). Rigorous coupled-wave analysis of metallic surface-relief gratings. $J$. Opt. Soc. Am. A, 11,1780-1787

Nylander, C., Liedberg, B., \& Lind, T. (1982). Gas detection by means of surface plasmon resonance. Sensors and Actuators, 3, 79-88. 